\title{
Integration of Operation Technology (OT) and Information Technology (IT) Through Intelligent Automation in Manufacturing Industries
}

\author{
ELAMVAZHUTHI KUPPUSAMY ${ }^{\mathrm{a}, 1}$, KAILASH MARIAPPAN $^{\mathrm{b}}$ \\ ${ }^{a}$ Founder \& CEO, YesLee Consultants \& YesLee Exports, Salem, Tamilnadu, India \\ ${ }^{\mathrm{b}}$ Founder \& CEO, KioTeckDigi Networks LLC(USA) \& KioTekDigi Networks Pvt Ltd., \\ India
}

\begin{abstract}
The results of integrating OT and IT in Manufacturing Industries are Increase in Productivity, Reduction in Waste, Savings in Labor and Energy and Better Maintenance. The convergence of IT and OT in IoT has been going on for a while and there isn't a strict division between them in the real world. Traditionally, IT is responsible for creating, storing and securing an organization's data. At the same time, OT focuses primarily on processes that take place in the physical world-think managing productivity, people, and machinery. There are Pre Design Phases and Final Design Phases for Implementation of the Integration process. Under the Pre Design Phases, Identify the types of Assets in Industrial Zone and those that support Production and then Identify "Who" owns the hardware and software in the asset. In the final Phases of implementation we have:

Requirements Phase: Interview all the system owners to gather requirements for operations, configuration and maintenance. Architectural Phase: Produce High level documentation and drawings to meet every requirement. Technical Design Phase: Produce detailed documentation such as drawings, switch configuration and VLAN, IP Address and Firewall ACLs. Implementation Phase: VERIFY "was the product built right?" and VALIDATE "was the right product built?" process. Maintain Phase: Modify configurations and assets to fix anomalies or required operational changes. The Intelligent Automation is Transforming Manufacturing Processes. The explosive growth of the cloud has made on - demand processing more accessible, more efficient and relatively lower cost. Robotic Process Automation (RPA) tools use Cognitive capabilities will replace those that don't.

There are several obvious benefits of automation that can be found in various automation projects as primary positive results. Among others, they include: \# Cost Reduction \# Higher Accuracy \# Increased focus on core competencies \# Improved productivity \# Better compliance \# Creating New jobs \# Reducing Employee turnover. Three types of automation in production can be distinguished: 1. Fixed automation, 2. Programmable automation, and 3. Flexible automation. In many industries IT and OT convergence already happens since quite some time (Oil and Gas is just one of the many). Utilities are realizing that to reap the full benefits of advanced metering and smart grid systems, IT and OT must work together. The convergence of IT and OT is about systems, standards and a new way of thinking. We are in the start of Industry 4.0, the industrial internet, cyber - physical systems and evolutions in areas and markets such as Building Management systems, smart metering and critical power.
\end{abstract}

${ }^{1}$ Corresponding Author. drkelamvazhuthi@gmail.com 
Keywords. Operation Technology (OT), Information Technology (IT), Convergence, Integration, Intelligent Automation (IA), manufacturing industries, Industry 4.0, IoT, Robotic Process Automation (RPA)

\section{Introduction on Intelligent Automation (IA) and how IA is Transforming Manufacturing Processes}

Intelligent Automation (IA) is the umbrella under which Artificial Intelligence (including machine learning and deep learning technologies and robotic process automation (including workflows) fall. Machine learning and deep Learning can be used to solve operational business problems, such as predictive analytics and predictive maintenance. Manufacturers already incorporate work flow design into their business operations, with many having workflows that are partially automated and save time for end- users.

By using date they are already recording, manufacturers can use predictive analytics to solve new problems and facilitate product engineering. Machine learning models are being used to predict how to fabricate specific physical materials and minimize anomalies in complex chemical reactions.

Predictive maintenance solutions allow machines report back when they will require servicing, thus reducing unplanned downtime and kicking off workflows to plan service.

Another area where Intelligent Automation is improving the experience for employees and customers alike is through chat bots and custom smart assistants. By connecting them to workflows and knowledge bases, they can help process orders, onboard employees and function as a specialized resource both in the operations room and on the factory floor.

\subsection{Intelligent Automation (IA) and use cases across Industries}

1.1.1 Intelligent Automation in Manufacturing- IA has triggered industrial revolutions - Industry 4.0 and Industry 5.0. These systems are inspired by recent technological advances, such as AI, ML, IOT, robotics, autonomous vehicles, 3D printing, AR and VR, wearables, additive manufacturing, nanotechnology, biotechnology, energy storage and quantum computing. As a result, physical and digital robot that are driven by cutting-edge Intelligent Automation technologies and tools can perform many operations, replacing and supplementing humans, and do so in the following ways:

- Automated factory floor: Physical robots and cobots (collaborative robots) can perform almost any manufacturing process in a factory. The factory floor has evolved over time into dark or lights-out premises where human participation is not required.

- Automated work flow: With digital bots, manufacturers can process huge amounts of data to streamline ordering, procurement, appointment scheduling and alerting.

- Predictive analytics: The capability to fix aberrations in a proactive manner helps manufacturers prevent outage and downtime. With predictive analytics 
solutions, maintenance engineers can anticipate errors and can address them before the equipment gets seriously affected.

- Machine Vision: This technology enables through quality inspection that is much more detailed and reliable than that performed by humans.

1.1.2 Intelligent Automation in Commerce: Some examples of IA in commerce are guidance through online and offline shopping, improved warehouse logistics, transaction security, data-driven planning of supply and delivery and hands-free checkout.

1.1.3 Intelligent Automation in Logistics and Transportation: Some major examples are route optimization, supply planning, autonomous cars and unmanned delivery.

1.1.4 Intelligent Automation in Healthcare: Healthcare providers are major consumers of IA. It enables caregivers to perform better the tasks of personalized treatment, predictive diagnosing, medical imaging, surgical operations, nursing, patient assistance, virtual consultations, remote monitoring and research and drug discovery.

1.1.5 Intelligent Automation in Telecom: The Telecom industry makes the most of IA to improve the efficiency of processes in the business areas of customer account management, Call center and Network operation.

\section{Ways Industries benefit from OT - IT Convergence}

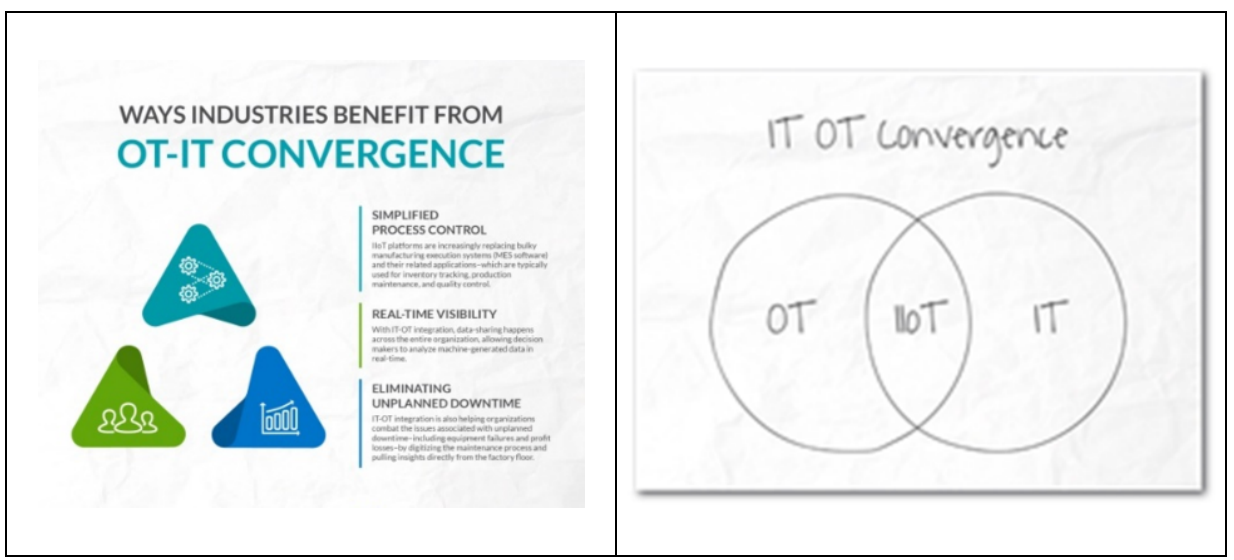

Figure 1. OT - IT Convergence.

Industrial Internet of Things (IIoT) generated unimaginable amounts of data collected from sensors, software, and large assets that could be spread across several locations. With lots of moving parts involved, OT - IT convergence gives organizations a strong foundation for improving internal processes, business decisions, and productivity. Here are some of the IT-OT convergence benefits manufacturing companies can expect: 
- Simplified Process Control: IIoT platforms are increasingly replacing bulky manufacturing execution systems (MES Software) and their related applications- which are typically used for inventory tracking, production maintenance, and quality control. These activities are distributed across both OT and IT, so when each department runs as separate entity, there's a missed opportunity to control and optimize manufacturing processes.

- Real Time Visibility: With IT - OT integration, data sharing happens across the entire organization, allowing - decision makers to analyze machine generated data in real - time. In the past, aggregating data from various machines, sensors, databases, and gateways was a manual effort that required compiling data from disparate sources and attempting to analyze that information as a single unit.

- Eliminating Unplanned Downtime: IT-OT integration is also helping organizations combat the issues associated with unplanned downtimeincluding equipment failures and profit loses - by digitizing the maintenance process and pulling insights directly from the factory floor. By monitoring machine performance, OT and IT teams can monitor equipment for early signs of trouble, and act accordingly.

\section{Technologies make IT - OT convergence possible}

Here are a few innovations making convergence a reality for more manufacturers:

- No Code Applications: No Code or Low code applications are used to connect IIoT devices, sensors and machines, as well as the humans that work with them on the factory floor. These programs are designed for human users without a background in coding, offering OT teams to create applications that work for the assembly line without having to learn how to code.

- Digital Twins: Digital twin software allows organizations to build virtual replicas of physical objects like heavy machinery or entire manufacturing systems in a digital environment. This technology allows manufacturers to see how environmental factors, changes to production cycle, and adverse events might impact product quality or productivity.

- Augmented Reality (AR): Augmented Reality tools aren't exactly at the forefront of innovations today - after all, Caterpillar has been using AR for inspections since 2015. Organizations can use AR in a connected system to oversee factory operations remotely, provide enhanced training simulations, and guide technicians through complex repairs.

- Edge Computing: The edge is increasingly considered to be the intersection where OT and IT meet. In the typical IIoT system, data is generated and collected by OT before sending it along to IT for analysis in the data center or the cloud. Edge Computing allows organizations to process data in real-time, rather than waiting for it show up in the cloud, allowing organizations to convert machine-generated data into actionable insights quickly. 


\section{Sharing the Factory Floor Data- Few proven approaches}

- Sensor to cellular to cloud- simple * Sensor to internet to cloud * Sensor to edge to cloud

- $\quad$ Through iDMZ ( Industrial Demilitarized Zone) - Complex

\section{Benefits of Converged OT and IT}

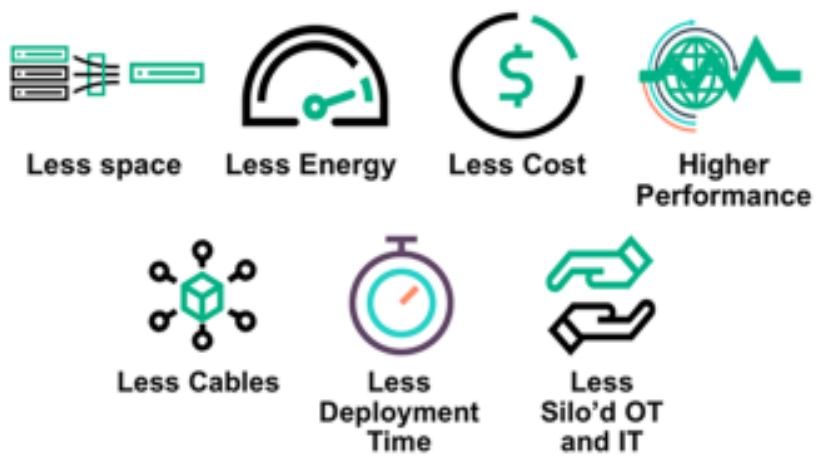

Figure 2. Benefits of Converged OT and IT.

\section{Phases of implementation:}

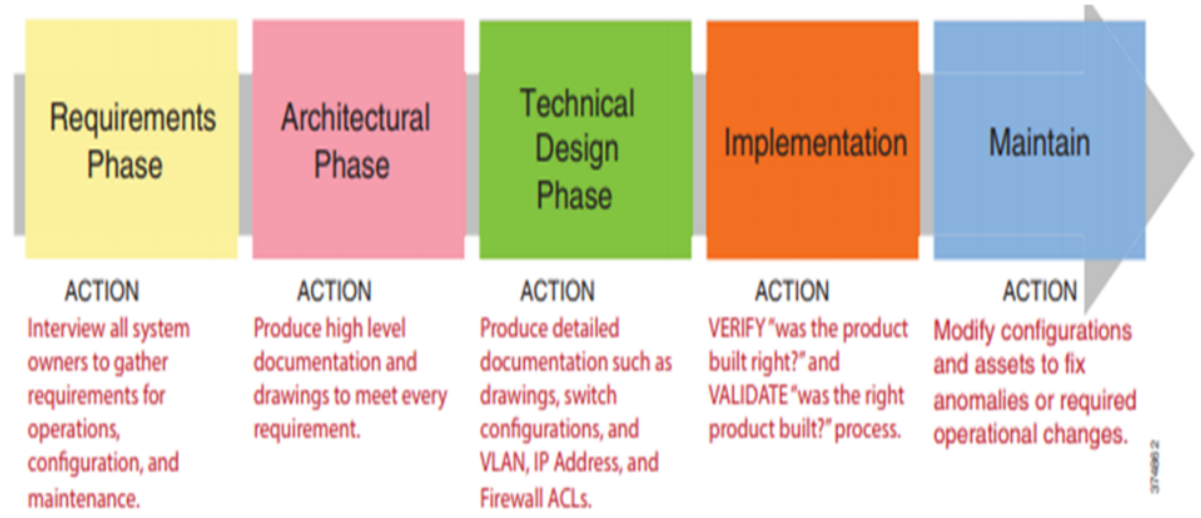

Figure 3. Phases of Implementation. 


\section{Overview of the integrated OT and IT systems:}

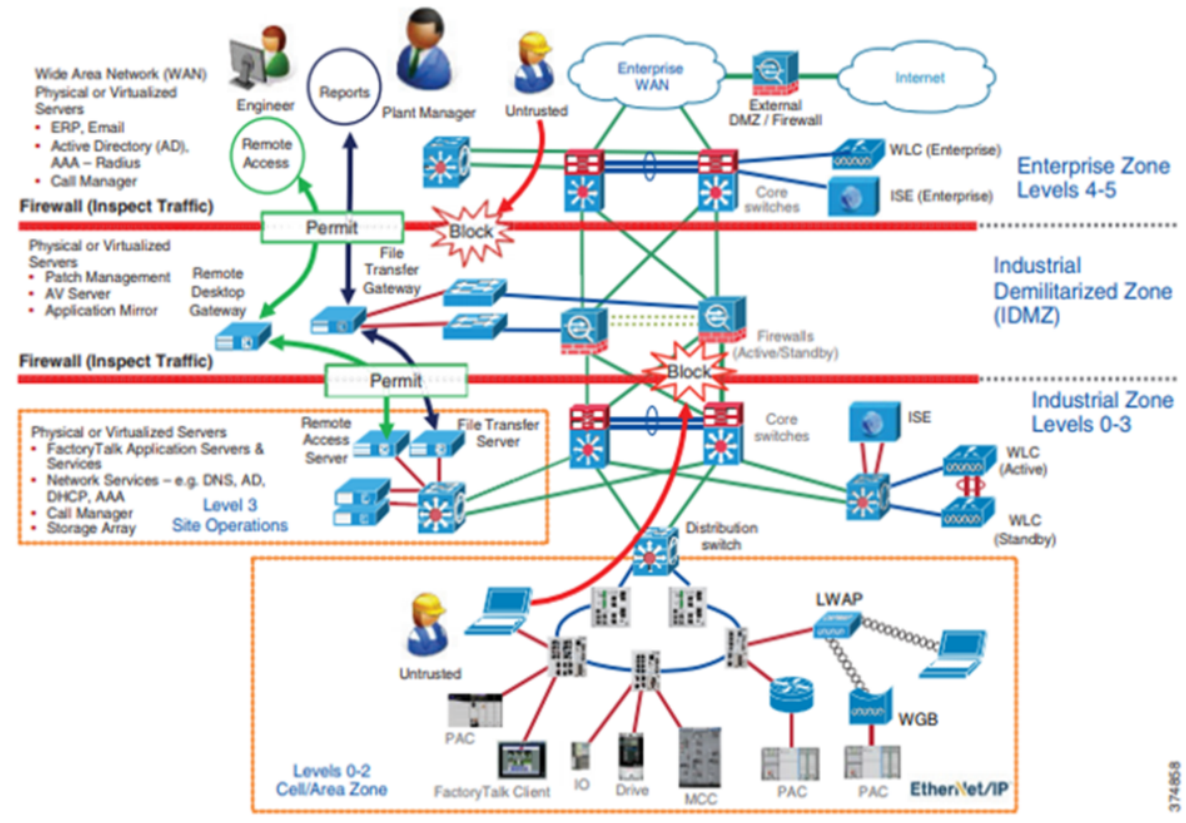

Figure 4. Overview of Integrated OT and IT Systems.

\section{Challenges of OT / IT Convergence:}

Digital transformation requires organizations to reform most or all areas of the organization to be successful. OTs modernization through IT integration is no different. Other challenges include:

- Process Convergence- Organizations may struggle with reorganizing previously siloed IT and OT departments to manage newly converged technology.

- Secure IoT Implementation- often, IoT initiatives are not owned by any one department and communication of a new project may not reach all the departments, especially in a more siloed organization. Typically OT departments have limited knowledge of security, and IT has a limited knowledge of the projects currently underway. This can create a dangerous security gap.

- Training- only recently have certifications like The Cisco certified Network Associate Industrial IoT been offered to help OT workers understand how they are accustomed to intersects with networked technology.

- Integration with existing systems- the business side of an organization may be tempted to replace technologies instead of modernizing existing OT with IT technology. This defeats one of the benefits of convergence, which is cost efficiency. The point of convergence is to do more with what is available. 


\section{Conclusion}

IT-OT convergence might seem overwhelming. However, it's an essential first step for organizations that wants to realize the benefits of IIoT. While uniting the two departments who have historically done their own thing comes with a whole range of challenges, benefits like streamlined processes, interoperability, and collaborative decision-making make IT-OT convergence well worth the effort.

\section{References}

[1] https://www.sam-solutions.com/blog/intelligent-automation/\#

[2] Pasca Bornet, Ian Barkin, Jochen Wirtz, Intelligent Automation, Amazon , Asia- Pacific Holdings Private Ltd., 2020

[3] https://www2.deloitte.com/uk

[4] https://www.i-scoop.eu/internet-of-things-guide/industrial-internet-things-it-ot/

[5] https://www.tiempodev.com/blog/it-ot-convergence-benefits-and-challenges-in-manufacturing/

[6] https://www.cio.com/article/3199019/cios-key-to-bridging-the-it-ot-divide.html 